\title{
Moments of the Wigner delay times
}

\author{
Gregory Berkolaiko ${ }^{1}$ and Jack Kuipers ${ }^{2}$ \\ ${ }^{1}$ Department of Mathematics, Texas A\&M University, College Station, TX \\ 77843-3368, USA \\ ${ }^{2}$ Institut für Theoretische Physik, Universität Regensburg, D-93040 Regensburg, \\ Germany \\ E-mail: Jack.Kuipers@physik. uni-regensburg.de
}

\begin{abstract}
The Wigner time delay is a measure of the time spent by a particle inside the scattering region of an open system. For chaotic systems, the statistics of the individual delay times (whose average is the Wigner time delay) are thought to be well described by random matrix theory. Here we present a semiclassical derivation showing the validity of random matrix results. In order to simplify the semiclassical treatment, we express the moments of the delay times in terms of correlation functions of scattering matrices at different energies. In the semiclassical approximation, the elements of the scattering matrix are given in terms of the classical scattering trajectories, requiring one to study correlations between sets of such trajectories. We describe the structure of correlated sets of trajectories and formulate the rules for their evaluation to the leading order in inverse channel number. This allows us to derive a polynomial equation satisfied by the generating function of the moments. Along with showing the agreement of our semiclassical results with the moments predicted by random matrix theory, we infer that the scattering matrix is unitary to all orders in the semiclassical approximation.

PACS numbers: 03.65.Sq, 05.45.Mt
\end{abstract}

\section{Introduction}

The dynamics of an open quantum system can be described by its scattering matrix. The scattering matrix is defined as the linear operator which transforms an incoming wavefunction, expanded in an asymptotic channel eigenmode basis, into the outgoing wavefunction. Probability conservation forces the scattering matrix to be unitary. As the scattering matrix describes the system, it can be used to investigate the desired physical properties of the system.

A quantity of particular interest is the Wigner time delay [1, 2] which was first derived for the one channel case from a Hermitian operator based on the scattering amplitude. The time delay is, as the name suggests, a measure of the extra time a particle spends inside the scattering region as a result of being scattered (for some details see [3] for example), and was later generalised to multi-channel scattering matrices [4]. With $M$ scattering channels, the Hermitian operator admits $M$ eigenvalues which are the individual delay times of the system, and the Wigner time delay is simply the average of these values.

For classically chaotic systems with a small opening, the probability for particles to remain inside the system decays exponentially with a typical time scale depending on the size of the opening. The exponential decay can be seen as a natural consequence of the 
ergodicity of the classical motion as (for reasonable times) the particle is equally likely to hit any part of the boundary of the system leading to a roughly constant small probability to leave through the opening each time it hits. The continuous limit of this process is the exponential decay, and the time scale associated to the decay is, for chaotic systems, exactly the average time delay [5] of the corresponding quantum system. Staying with the classical version for now, if we spread particles evenly over the available space and evolve them over time they will condense onto a typically fractal pattern arranged around the zero-measure set of trapped periodic orbits that never leave the system. From this set, and following their stable manifolds very closely we can construct trajectories that start outside the system, approach trapped orbits and remain inside the system for arbitrarily long times before eventually escaping following the unstable manifolds. In fact, it turns out that these trajectories, or rather correlations between them, are responsible in the semiclassical limit for recreating the oscillating part of the time delay [6], which is given exactly in terms of the trapped periodic orbits [7, 8].

As the oscillating part of the time delay is given in terms of periodic orbits in the semiclassical limit (of $\hbar \rightarrow 0$ ), correlations between periodic orbits must then be responsible for the typical fluctuations of the Wigner time delay. In particular the form factor of the time delay can be written in terms of pairs of periodic orbits and the types of correlations that contribute were first treated for the spectral form factor for closed systems, where Gutzwiller's trace formula [9, 10] likewise provides a sum over pairs of orbits. The semiclassical treatment of this sum started with the diagonal approximation [11] of pairing orbits with themselves (or their time reversal) and used the sum rule of [12] to find the semiclassical contribution. This was followed more recently by the treatment of correlated pairs of orbits which are almost identical everywhere, but which differ in a very small region called an encounter where the orbits behave differently and end up reconnecting inside the encounter [13. All the possible types of orbits with encounters were then generated and treated [14, 15], and this treatment was applied to the time delay to obtain a semiclassical expansion for its form factor [16].

The expansion for the form factor of the Wigner time delay was shown to agree with the result from random matrix theory (RMT), in line with the idea that most properties of quantum chaotic systems are well described by the results of RMT. But RMT can tell us at lot more about the expected typical behaviour of the individual delay times, and in particular that their distribution should have a compact support [17] in the limit where the opening supports a large number of channels. Of course the classical decay gives no upper bound on the distribution of the delay times, and so (if we expect to recover RMT results) quantum interference as expressed through semiclassical correlations between classical trajectories should somehow contrive to limit the maximum delay time.

Our aim in this article is to identify the sort of correlations that contribute in the semiclassical limit and to describe how they lead to the RMT result. We start with an introduction to the time delay in section 2. To study the distribution of the delay times we evaluate their moments which can be expressed in terms of correlation functions of the scattering matrix. This approach simplifies the semiclassical treatment compared to the direct evaluation of the time delay matrix.

In section 2.2 we briefly review the RMT results before applying the semiclassical approximation to the scattering matrix elements to obtain expressions in terms of scattering trajectories. For the low order moments, which we treat in section 3, we build on the work of the semiclassical treatment of the conductance [18, 19] and its second moment [20, 21]. To treat all the moments, we delve into the combinatorial relations that first arose in the treatment of the moments of the transmission amplitudes [22]. This 
work is extended in section 4 , where we find an implicit expression for the generating function of the moments and show complete agreement with the RMT distribution of delay times. Considering a simpler case in Appendix A, we briefly derive the correlation functions which recently appeared in the semiclassical treatment of the density of states of Andreev billiards [23.

\section{The time delay matrix}

If we consider a chaotic cavity with one or more open leads that carry $M$ scattering channels, the scattering dynamics is encoded in the $M \times M$ unitary scattering matrix $S(E)$ which relates the incoming and outgoing waves. We are interested in the Wigner time delay, which represents the extra time spent in the scattering process compared to free motion, and which can be found using the Wigner-Smith matrix [1, 2, 4]

$$
Q=\frac{\hbar}{\mathrm{i}} S^{\dagger}(E) \frac{\mathrm{d} S(E)}{\mathrm{d} E} .
$$

Differentiating the unitarity condition, $S^{\dagger}(E) S(E)=I$, with respect to $E$, we see that

$$
S^{\dagger}(E) \frac{\mathrm{d} S(E)}{\mathrm{d} E}=-\frac{\mathrm{d} S^{\dagger}(E)}{\mathrm{d} E} S(E),
$$

and that the matrix $Q$ is Hermitian with real eigenvalues. The $M$ eigenvalues are the individual delay times of the system, and the average value of these times is the Wigner time delay

$$
\tau_{\mathrm{W}}(E)=\frac{1}{M} \operatorname{Tr}[Q]
$$

The moments of the eigenvalues are given by

$$
m_{n}=\frac{1}{M} \operatorname{Tr}\left[Q^{n}\right],
$$

and can be used to recover the complete distribution of the eigenvalues of $Q$.

We can also obtain the Wigner time delay from a correlation function of the scattering matrix

$$
C(\epsilon)=\frac{1}{M} \operatorname{Tr}\left[S^{\dagger}\left(E-\frac{\epsilon \hbar \mu}{2}\right) S\left(E+\frac{\epsilon \hbar \mu}{2}\right)\right],
$$

where it is convenient to specify the energy difference in units of $\hbar \mu$, where $\mu$ is the classical escape rate of the system. This correlation function provides a symmetrized version of the time delay [24]

$$
\tau_{\mathrm{W}}=\left.\frac{1}{\mathrm{i} \mu} \frac{\mathrm{d}}{\mathrm{d} \epsilon} C(\epsilon)\right|_{\epsilon=0}=\frac{\hbar}{2 \mathrm{i} M} \operatorname{Tr}\left[S^{\dagger}(E) \frac{\mathrm{d} S(E)}{\mathrm{d} E}-\frac{\mathrm{d} S^{\dagger}(E)}{\mathrm{d} E} S(E)\right],
$$

which agrees with the definition in (3) because of the unitarity of the scattering matrix as expressed through (2).

As well as giving us the first moment of the delay times (the Wigner time delay), this correlation function can also provide us with the second. Differentiating (2) again we obtain

$$
S^{\dagger}(E) \frac{\mathrm{d}^{2} S(E)}{\mathrm{d} E^{2}}+\frac{\mathrm{d}^{2} S^{\dagger}(E)}{\mathrm{d} E^{2}} S(E)=-2 \frac{\mathrm{d} S^{\dagger}(E)}{\mathrm{d} E} \frac{\mathrm{d} S(E)}{\mathrm{d} E},
$$

which can be used to simplify the second derivative of $C(\epsilon)$ to

$$
\left.\frac{\mathrm{d}^{2}}{\mathrm{~d} \epsilon^{2}} C(\epsilon)\right|_{\epsilon=0}=-\frac{\hbar^{2} \mu^{2}}{M} \operatorname{Tr}\left[\frac{\mathrm{d} S^{\dagger}(E)}{\mathrm{d} E} \frac{\mathrm{d} S(E)}{\mathrm{d} E}\right] .
$$


Inserting the identity matrix in the form $S(E) S^{\dagger}(E)$, we obtain

$$
\left.\frac{\mathrm{d}^{2}}{\mathrm{~d} \epsilon^{2}} C(\epsilon)\right|_{\epsilon=0}=\frac{(\mathrm{i} \mu)^{2}}{M} \operatorname{Tr}\left[Q^{\dagger} Q\right]=(\mathrm{i} \mu)^{2} m_{2},
$$

and the second moment of the delay times.

\subsection{Various approaches to higher moments}

Differentiating the correlation function $C(\epsilon)$ further cannot produce higher moments since the $n$-th moment involves the first derivative of $S(E)$ appearing $n$ times while the definition of $C(\epsilon)$, see (5), involves only two matrices. One way to resolve this difficulty is to define a higher correlation function

$$
C(\boldsymbol{\epsilon}, n)=\frac{1}{M} \operatorname{Tr}\left[\prod_{j=1}^{n} S^{\dagger}\left(E-\frac{\epsilon_{j} \hbar \mu}{2}\right) S\left(E+\frac{\epsilon_{j} \hbar \mu}{2}\right)\right],
$$

where $\boldsymbol{\epsilon}=\left(\epsilon_{1}, \ldots, \epsilon_{n}\right)$ is the vector of energy differences. From the $n$-th correlation function we can obtain all the moments up to the $(2 n)$-th by differentiating. The formulae, which are different for odd and even moments, are

$$
\begin{aligned}
& m_{2 k-1}=\left.\frac{1}{(\mathrm{i} \mu)^{(2 k-1)}}\left(\prod_{j=1}^{k-1} \frac{\mathrm{d}^{2}}{\mathrm{~d} \epsilon_{j}^{2}}\right) \frac{\mathrm{d}}{\mathrm{d} \epsilon_{k}} C(\boldsymbol{\epsilon}, n)\right|_{\boldsymbol{\epsilon}=0}, \\
& m_{2 k}=\left.\frac{1}{(\mathrm{i} \mu)^{2 k}}\left(\prod_{j=1}^{k} \frac{\mathrm{d}^{2}}{\mathrm{~d} \epsilon_{j}^{2}}\right) C(\boldsymbol{\epsilon}, n)\right|_{\epsilon=0} .
\end{aligned}
$$

Though this provides an efficient way to calculate the lower order moments, the different energy arguments complicate the semiclassical treatment. On the other hand, setting all energy arguments to $\epsilon$ and differentiating $n$ times leads to appearance of additional unwanted terms. To deal with such terms we define the correlation function

$$
D(\epsilon, n)=\frac{1}{M} \operatorname{Tr}\left[S^{\dagger}\left(E-\frac{\epsilon \hbar \mu}{2}\right) S\left(E+\frac{\epsilon \hbar \mu}{2}\right)-I\right]^{n} .
$$

Because of the relation

$$
\left.\frac{1}{n !} \frac{\mathrm{d}^{n}}{\mathrm{~d} \epsilon^{n}}[f(\epsilon)-f(0)]^{n}\right|_{\epsilon=0}=\left[f^{\prime}(0)\right]^{n},
$$

we can see that the moments of the time delay matrix are now given directly by

$$
m_{n}=\left.\frac{1}{(\mathrm{i} \mu)^{n} n !} \frac{\mathrm{d}^{n}}{\mathrm{~d} \epsilon^{n}} D(\epsilon, n)\right|_{\epsilon=0} .
$$

Expanding the $n$-th power in (13) we can also obtain the moments as

$$
m_{n}=\left.\frac{1}{(\mathrm{i} \mu)^{n} n !} \frac{\mathrm{d}^{n}}{\mathrm{~d} \epsilon^{n}} \sum_{k=1}^{n}(-1)^{n-k}\left(\begin{array}{l}
n \\
k
\end{array}\right) C(\epsilon, k)\right|_{\epsilon=0},
$$

in terms of the correlation functions

$$
C(\epsilon, n)=\frac{1}{M} \operatorname{Tr}\left[S^{\dagger}\left(E-\frac{\epsilon \hbar \mu}{2}\right) S\left(E+\frac{\epsilon \hbar \mu}{2}\right)\right]^{n} .
$$

The correlation functions $C(\epsilon, n)$ have applications outside the scope of this article (see [23]) and thus their semiclassical evaluation is a question of stand-alone interest. However, we found that performing the summation in (16) is a difficult task. Thus 
from now on we will work with $D(\epsilon, n)$ directly. However, the technique we develop for evaluating $D(\epsilon, n)$ is suitable for the simpler function $C(\epsilon, n)$ as well. We exploit it by deriving an equation for the generating function of $C(\epsilon, n)$ in Appendix $\mathrm{A}$

Finally, we note that one can use the semiclassical approximation directly in the definition of the Wigner-Smith matrix, equation (11). The approach we took, however, allows us to build on previous semiclassical work performed in open systems, in particular on the average conductance of a chaotic ballistic device and its moments 19, 20, 21, 22. We will obtain, as in the case of the conductance and shot noise, simple diagrammatic rules for the semiclassical contributions of correlated trajectories. But before approaching this task we will quickly review the RMT results for the delay times.

\subsection{Random matrix predictions}

The random matrix result for the probability distribution of the delay times is 17 .

$$
\rho(\tau)=\frac{1}{2 \pi \tau^{2}} \sqrt{\left(\tau_{+}-\tau\right)\left(\tau-\tau_{-}\right)}, \quad \tau_{ \pm}=\frac{3 \pm \sqrt{8}}{\mu},
$$

from which we can calculate the moments

$$
m_{n}=\int \tau^{n} \rho(\tau) \mathrm{d} \tau=\frac{1}{2 \pi} \int_{\tau_{-}}^{\tau_{+}} \tau^{n-2} \sqrt{\left(\tau_{+}-\tau\right)\left(\tau-\tau_{-}\right)} \mathrm{d} \tau .
$$

Substituting $t+3=\mu \tau$ this can be written more obviously in terms of the moments of the semicircle distribution

$$
m_{n}=\frac{2}{\mu^{n}} \int_{-R}^{R}(t+3)^{n-2} \frac{2 \sqrt{R^{2}-t^{2}}}{\pi R^{2}} \mathrm{~d} t,
$$

with $R^{2}=8$. The odd moments of the semicircle distribution are 0 , while the even moments can be written as

$$
\tilde{m}_{2 n}=\int_{-R}^{R} t^{2 n} \frac{2 \sqrt{R^{2}-t^{2}}}{\pi R^{2}} \mathrm{~d} t=\left(\frac{R}{2}\right)^{2 n} c_{n}=2^{n} c_{n},
$$

where $c_{n}$ are the Catalan numbers defined as

$$
c_{n}=\frac{1}{n+1}\left(\begin{array}{c}
2 n \\
n
\end{array}\right)=\frac{(2 n) !}{(n+1) ! n !} .
$$

By expanding the term $(t+3)^{n-2}$ in (20) in powers of $t$, we can therefore express the moments (beyond the first) by the following sums

$$
\begin{aligned}
& m_{2 n+2}=\frac{2}{\mu^{2 n+2}} \sum_{k=0}^{n}\left(\begin{array}{c}
2 n \\
2 k
\end{array}\right) 3^{2(n-k)} 2^{k} c_{k}, \\
& m_{2 n+3}=\frac{2}{\mu^{2 n+3}} \sum_{k=0}^{n}\left(\begin{array}{c}
2 n+1 \\
2 k
\end{array}\right) 3 \cdot 3^{2(n-k)} 2^{k} c_{k} .
\end{aligned}
$$

The sequence of integers obtained from combining the Catalan numbers as above, and hence appearing in the moments, can then be shown [25] to be the so-called Schröder numbers (Sloane's A006318 or $2 \times$ A001003) [26]. More importantly for our purposes, the Schröder numbers can be derived from a generating function [26] and so the moments of the delay times can also be generated from

$$
G(s)=\sum_{n=1} \mu^{n} s^{n} m_{n}=\frac{1-s-\sqrt{1-6 s+s^{2}}}{2} .
$$




\section{Calculation of the low correlation functions}

Since the matrix $S(E)$ is unitary we can rewrite the correlation function $D(\epsilon, n)$ as

$$
\begin{aligned}
D(\epsilon, n) & =\frac{1}{M} \operatorname{Tr}\left[S^{\dagger}\left(E-\frac{\epsilon \hbar \mu}{2}\right) S\left(E+\frac{\epsilon \hbar \mu}{2}\right)-S^{\dagger}(E) S(E)\right]^{n} \\
& =\frac{1}{M} \sum_{i_{1}, \ldots, i_{n}}\left(S_{-}^{\dagger} S_{+}-S^{\dagger} S\right)_{i_{1}, i_{2}}\left(S_{-}^{\dagger} S_{+}-S^{\dagger} S\right)_{i_{2}, i_{3}} \cdots
\end{aligned}
$$

where the summation in the second line is over choices of $n$ incoming channels and the \pm subscripts represent the different energy arguments of the scattering matrices. To evaluate $D(\epsilon, n)$ semiclassically, we will use the semiclassical approximation for the scattering matrix elements, which is given in terms of open trajectories [27, 28, 18]

$$
S_{b a}(E) \approx \frac{1}{\sqrt{T_{\mathrm{H}}}} \sum_{\zeta(a \rightarrow b)} A_{\zeta} \mathrm{e}^{\frac{i}{\hbar} S_{\zeta}}
$$

The $T_{\mathrm{H}}$ appearing in the prefactor is the Heisenberg time, and it is simply related to the classical escape rate by $\mu=M / T_{\mathrm{H}}$. In the sum which is over all classical trajectories $\zeta$ that start in channel $a$ and end in channel $b$ (where the channels fix the absolute value of the angles at which the trajectories enter and leave the cavity) $S_{\zeta}$ is the action of the trajectory $\zeta$ and $A_{\zeta}$ its stability amplitude [28 including the phase due to the number of conjugate points along the trajectory. As our starting point, we will substitute approximation (28) into (26), expand the action up to first order in energy $S_{\zeta}(E+\delta E) \approx S_{\zeta}(E)+T_{\zeta}(E) \delta E$, where $T_{\zeta}$ is the time the trajectory $\zeta$ spends inside the system, and ignore any change in the slowly varying prefactor $A_{\zeta}$.

Below we perform this calculation for $n=1,2$ and 3 before formulating the general counting rules and performing the evaluation of $D(\epsilon, n)$ for general $n$.

\subsection{Calculating $D(\epsilon, 1)$}

After semiclassical approximation (28), the correlation function $D(\epsilon, 1)$ becomes

$$
\begin{aligned}
D(\epsilon, 1) & =\frac{1}{M} \sum_{i_{1}}\left(S_{-}^{\dagger} S_{+}-S^{\dagger} S\right)_{i_{1}, i_{1}} \\
& \approx \frac{1}{M T_{\mathrm{H}}} \sum_{i_{1}, o_{1}} \sum_{\zeta, \zeta^{\prime}\left(i_{1} \rightarrow o_{1}\right)} A_{\zeta} A_{\zeta^{\prime}}^{*} \mathrm{e}^{\frac{\mathrm{i}}{\hbar}\left(S_{\zeta}-S_{\zeta^{\prime}}\right)}\left(\mathrm{e}^{\frac{\mathrm{i} \epsilon \mu}{2}\left(T_{\zeta}+T_{\zeta^{\prime}}\right)}-1\right),
\end{aligned}
$$

which is a sum over trajectory pairs $\zeta, \zeta^{\prime}$ both of which start and end in the same channels ( $i_{1}$ and $o_{1}$ respectively), followed by a sum over all the possible channels. We note that the difference of actions $S_{\zeta}-S_{\zeta^{\prime}}$ is divided by $\hbar \ll 1$ and therefore the resulting phase oscillates wildly unless the action difference is of order $\hbar$. The semiclassical expansion is based on identifying couples (or, more generally, families) of orbits that have small action differences.

To leading order in inverse channel number $1 / M$ the first correlation function can be calculated as in [6] using the diagonal approximation [11. This approximation restricts the sum to trajectories $\zeta$ and $\zeta^{\prime}$ that are identical

$$
D^{\text {diag }}(\epsilon, 1)=\frac{1}{M T_{\mathrm{H}}} \sum_{i_{1}, o_{1}} \sum_{\zeta\left(i_{1} \rightarrow o_{1}\right)}\left|A_{\zeta}\right|^{2}\left(\mathrm{e}^{\mathrm{i} \epsilon \mu T_{\zeta}}-1\right) .
$$


The sum in (30) can be performed by using a sum rule for open trajectories [18] which turns it into an integral over the trajectory time $T$

$$
\sum_{\zeta\left(i_{1} \rightarrow o_{1}\right)}\left|A_{\zeta}\right|^{2} \ldots \approx \int_{0}^{\infty} \mathrm{d} T \mathrm{e}^{-\mu T} \ldots
$$

The exponential term in (31) represents the average probability that a trajectory remains in the system for the time $T$, while the sum over channels, where we can pick both $i_{1}$ and $o_{1}$ from the $M$ possible channels, simply gives a factor of $M^{2}$. The diagonal approximation thus gives

$$
\begin{aligned}
D^{\mathrm{diag}}(\epsilon, 1) & \approx \frac{M}{T_{\mathrm{H}}} \int_{0}^{\infty} \mathrm{d} T\left(\mathrm{e}^{-\mu(1-\mathrm{i} \epsilon) T}-\mathrm{e}^{-\mu T}\right) \\
& =\frac{1}{1-\mathrm{i} \epsilon}-1=\frac{\mathrm{i} \epsilon}{1-\mathrm{i} \epsilon},
\end{aligned}
$$

where we have used the fact that $\mu=M / T_{\mathrm{H}}$ to simplify. Substituting into (15) we obtain

$$
\tau_{\mathrm{W}}=\frac{1}{\mu}
$$

which just states that the average delay time is the inverse of the classical escape rate as we might expect.

\subsection{Calculating $D(\epsilon, 2)$}

To obtain the next moment we move to the correlation function $D(\epsilon, 2)$. For this we will need to treat not only the diagonal pairs, but also correlated trajectories that have encounters. The calculation follows the calculation of the shot noise power [20] which was first performed to leading order in inverse channel number for quantum graphs 29. The semiclassical treatment of the shot noise builds on work on the conductance [18, 19, which itself is built on the work on spectral statistics [13, 14, 15]. $S$

First we slightly modify (27) to explicitly include an indication of the unitarity of

$D(\epsilon, 2)=\frac{1}{M} \sum_{i_{1}, i_{2}}\left(S_{-}^{\dagger} S_{+}-\delta_{i_{1}, i_{2}} S^{\dagger} S\right)_{i_{1}, i_{2}}\left(S_{-}^{\dagger} S_{+}-\delta_{i_{1}, i_{2}} S^{\dagger} S\right)_{i_{2}, i_{1}}$.

While the semiclassical approximation (28) preserves the unitarity of $S$, and thus the Kronecker deltas are not necessary, their inclusion greatly facilitates the derivation.

We now write down the semiclassical expression for the correlation function $D(\epsilon, 2)$ in terms of open trajectories

$$
\begin{aligned}
D(\epsilon, 2) \approx \frac{1}{M T_{\mathrm{H}}^{2}} & \sum_{\substack{i_{1}, i_{2} \\
o_{1}, o_{2}}} \sum_{\substack{\zeta\left(i_{1} \rightarrow o_{1}\right) \\
\zeta^{\prime}\left(i_{2} \rightarrow o_{1}\right)}} \sum_{\substack{\xi\left(i_{2} \rightarrow o_{2}\right) \\
\xi^{\prime}\left(i_{1} \rightarrow o_{2}\right)}} A_{\zeta} A_{\zeta^{\prime}}^{*} A_{\xi} A_{\xi^{\prime}}^{*} \mathrm{e}^{\frac{\mathrm{i}}{\hbar}\left(S_{\zeta}-S_{\zeta^{\prime}}+S_{\xi}-S_{\xi^{\prime}}\right)} \\
& \times\left(\mathrm{e}^{\frac{\mathrm{i} \epsilon \mu}{2}\left(T_{\zeta}+T_{\zeta^{\prime}}\right)}-\delta_{i_{1}, i_{2}}\right)\left(\mathrm{e}^{\frac{\mathrm{i} \epsilon \mu}{2}\left(T_{\xi}+T_{\xi^{\prime}}\right)}-\delta_{i_{1}, i_{2}}\right) .
\end{aligned}
$$

For diagonal terms we can either pair $\zeta=\zeta^{\prime}$ and $\xi=\xi^{\prime}$, or $\zeta=\xi^{\prime}$ and $\xi=\zeta^{\prime}$. For the first case, the start channels $i_{1}$ and $i_{2}$ must coincide thus triggering the Kronecker delta. For the second case we have $o_{1}=o_{2}$. Either case leads to a (leading order) channel 


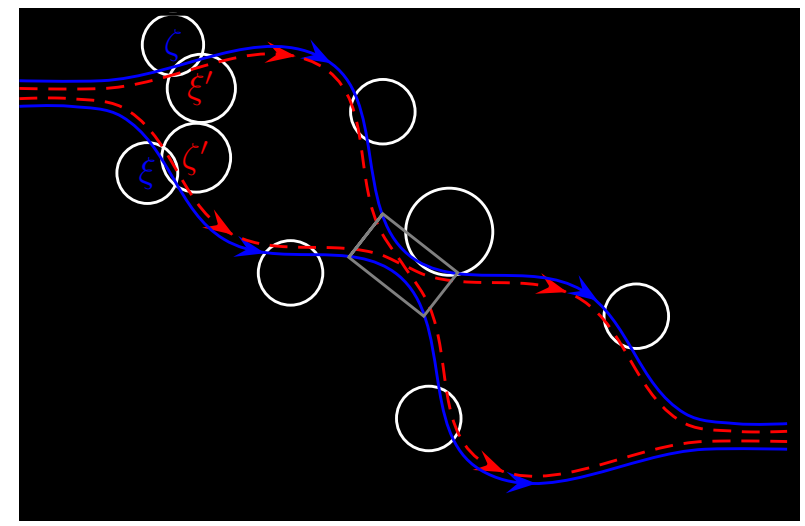

Figure 1. An example of two trajectories with a single encounter and two partner trajectories.

factor of $M^{3}$, because the number of channels we can choose in the outer summation is 3. Putting these two possibilities into (36) we can write the diagonal contribution as

$$
\begin{aligned}
D^{\text {diag }}(\epsilon, 2)= & \frac{M^{2}}{T_{\mathrm{H}}^{2}} \sum_{\zeta, \xi}\left|A_{\zeta}\right|^{2}\left|A_{\xi}\right|^{2}\left(\mathrm{e}^{\mathrm{i} \epsilon \mu T_{\zeta}}-1\right)\left(\mathrm{e}^{\mathrm{i} \epsilon \mu T_{\xi}}-1\right) \\
& +\frac{M^{2}}{T_{\mathrm{H}}^{2}} \sum_{\zeta, \xi}\left|A_{\zeta}\right|^{2}\left|A_{\xi}\right|^{2} \mathrm{e}^{\mathrm{i} \epsilon \mu T_{\zeta}} \mathrm{e}^{\mathrm{i} \epsilon \mu T_{\xi}} .
\end{aligned}
$$

Note that in the second case it can also happen that $i_{1}=i_{2}$, but this case gives a lower order channel factor of $M^{2}$ and is therefore neglected. Using the open sum rule from (31) we get

$$
D^{\text {diag }}(\epsilon, 2)=\frac{(\mathrm{i} \epsilon)^{2}}{(1-\mathrm{i} \epsilon)^{2}}+\frac{1}{(1-\mathrm{i} \epsilon)^{2}} .
$$

However, as we know from the calculation of the shot noise [29, 20], the diagonal terms are not the only ones that contribute to leading order in inverse channel number. If the trajectories $\zeta$ and $\xi$ come very close to each other in an encounter, as in Figure1, then the partner trajectories can cross over inside the encounter leading to a quadruplet of trajectories with a small action difference. Such a quadruplet can then give a contribution in the semiclassical limit. While such an encounter makes the contribution higher order in inverse channel number, the sum over channels now contributes the factor of $M^{4}$. As a result the quadruplet contributes at the same order as the diagonal terms.

To calculate the contribution, we simply put the additional energy arguments into the calculation of the shot noise power. The contribution can be separated into a product over the links and the encounters [20] and written as

$$
\begin{aligned}
D^{\left(2^{1}\right)}(\epsilon, 2)= & \frac{M^{3}}{T_{\mathrm{H}}^{2}} \int_{0}^{\infty} \mathrm{d} t_{1} \mathrm{e}^{-\mu(1-\mathrm{i} \epsilon) t_{1}} \int_{0}^{\infty} \mathrm{d} t_{2} \mathrm{e}^{-\mu(1-\mathrm{i} \epsilon) t_{2}} \\
& \times \int_{0}^{\infty} \mathrm{d} t_{3} \mathrm{e}^{-\mu(1-\mathrm{i} \epsilon) t_{3}} \int_{0}^{\infty} \mathrm{d} t_{4} \mathrm{e}^{-\mu(1-\mathrm{i} \epsilon) t_{4}} \\
& \times \int \mathrm{d} s \mathrm{~d} u \frac{\mathrm{e}^{-\mu(1-2 \mathrm{i} \epsilon) t_{\text {enc }}(s, u)} \mathrm{e}^{\frac{1}{\hbar} s u}}{\Omega t_{\text {enc }}(s, u)}
\end{aligned}
$$


where $\left(2^{1}\right)$ refers to the structure of the diagram: one encounter with two (unprimed) trajectories meeting (a "2-encounter"). In the final integral, $s$ and $u$ are the separations along the stable and unstable manifolds of the two original stretches inside the encounter and $\Omega$ is the volume of the available phase space, while $t_{\mathrm{enc}}(s, u)$ is the duration of the encounter. An important point is that although the encounter involves two trajectory stretches, as they are close to each other they will either remain inside the system or escape together and their average survival probability is given by the time of just a single stretch. The presence of the encounter actually slightly enhances the survival probability of the whole trajectory quadruplet, and this tiny classical effect has important semiclassical implications. Elsewhere in (39), the $t_{i}$ are the durations of the link stretches as depicted in Figure 1]. Performing the integrals following [20, we obtain the result

$$
D^{\left(2^{1}\right)}(\epsilon, 2)=\frac{-(1-2 \mathrm{i} \epsilon)}{(1-\mathrm{i} \epsilon)^{4}},
$$

The structure of the answer is very simple: each $l$-encounter contributes a factor of $-(1-\mathrm{i} l \epsilon)$, while each link stretch gives the factor $(1-\mathrm{i} \epsilon)^{-1}$. These diagrammatic rules, which first arose for the conductance [19], massively simplify calculating the semiclassical contributions, and are the reason we consider correlation functions rather than the time delay directly.

We can combine the two leading order results from (38) and (40) to obtain the second moment

$$
m_{2}=\frac{2}{\mu^{2}}
$$

In conclusion of this subsection we mention that the configurations described as diagonal contributions above can be obtained from the diagram in Figure 1 by setting $t_{1}=t_{3}=0$ for the first case and $t_{2}=t_{4}=0$ for the second. We will refer to this reduction as moving or sliding an encounter into the lead. As we have seen above, moving an encounter into the input lead can lead to a contribution that is different from the encounter in the output lead.

\subsection{Calculating $D(\epsilon, 3)$}

Before we proceed to calculate $D(\epsilon, 3)$, we will briefly look at how we can form the diagrams that contribute. As we have seen, the $n$-th correlator is expressed as a sum over $2 n$ trajectories

$$
D(\epsilon, n) \approx \frac{1}{M T_{\mathrm{H}}^{n}} \sum_{\left\{i_{j}, o_{j}\right\}} \sum_{\substack{\left\{\zeta_{j}\left(i_{j} \rightarrow o_{j}\right)\right\} \\\left\{\zeta_{j}^{\prime}\left(i_{j+1} \rightarrow o_{j}\right)\right\}}} \prod_{j=1}^{n} A_{\zeta_{j}} A_{\zeta_{j}^{\prime}}^{*} \mathrm{e}^{\frac{i}{\hbar}\left(S_{\zeta_{j}}-S_{\zeta_{j}^{\prime}}\right)}\left(\mathrm{e}^{\frac{\mathrm{i} \epsilon \mu}{2}\left(T_{\zeta_{j}}+T_{\zeta_{j}^{\prime}}\right)}-\delta_{i_{j}, i_{j+1}}\right) .
$$

Taking the trace of the product of matrices means that we identify $i_{n+1}=i_{1}$. Therefore the trajectories complete a cycle, if we consider moving forward along the unprimed trajectories and back along the primed ones. The resulting structure for $n=3$ is shown in Figure $2 \mathrm{a}$ and, as we have also seen, in (42) we add the actions of the unprimed trajectories and subtract the actions of the primed ones, so the resulting phase oscillates wildly unless the total action difference is of the order of $\hbar$.

To obtain such a small action difference we can collapse all the trajectories onto each other, as in Figure 2 $\mathrm{b}$, creating encounters of the type we saw in Figure 1. It turns out that to obtain all contributions from this type of direct collapse we need to 
a)

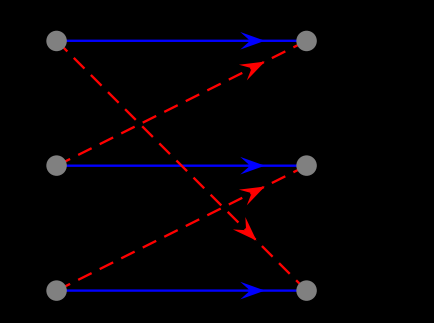

d)

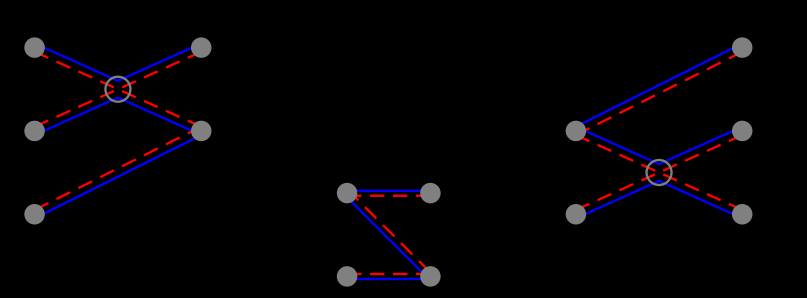

b)

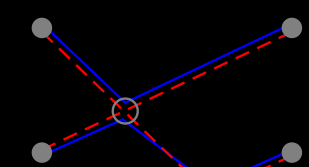

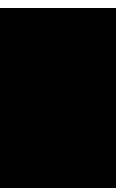

)

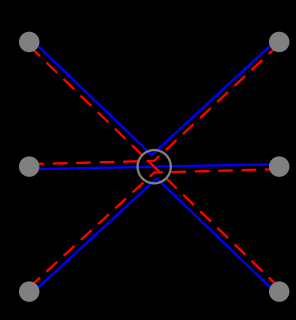

)

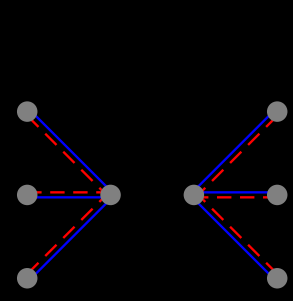

Figure 2. The original trajectory structure for $D(\epsilon, 3)$ in a) can be collapsed down to (three copies) of the structure in b), which has two 2-encounters, to ensure a small action difference. Sliding the encounters together creates the single 3-encounter in c), while moving encounters into the leads generates all the further possibilities. Trajectories $\zeta_{j}$ are indicated by solid lines and trajectories $\zeta_{j}^{\prime}$ by dashed lines.

cyclically permute the labels of the trajectories, resulting in three copies of the diagram in Figure 2b. Alongside this direct collapse we can imagine sliding the encounters together to create a single diagram with a single 3-encounter, as shown in Figure 2r. Further possibilities then arise from sliding the encounters into the leads, giving the remaining diagrams depicted in Figure 2 .

Before we write down the total contribution, we consider two instructive examples. First we evaluate the contribution from the diagram in Figure 22. We assume the trajectories indicated by solid lines are numbered top to bottom as $\zeta_{1}, \zeta_{2}$ and $\zeta_{3}$. Then we have $i_{1}=i_{2}$ since the first encounter is in the incoming lead. This activates one of the Kronecker deltas in the expression for $D(\epsilon, 3)$ (see (42) or the similar expression in (35)). The terms due to trajectories $\zeta_{2}$ and $\zeta_{3}$, which have a non-degenerate encounter, are the same as in (39), giving in total

$$
\begin{aligned}
D_{i_{1}=i_{2}}^{\left(2^{2}\right)}(\epsilon, 3)= & \frac{M^{4}}{T_{\mathrm{H}}^{3}} \int_{0}^{\infty} \mathrm{d} T_{\zeta_{1}} \mathrm{e}^{-\mu T_{\zeta_{1}}}\left(\mathrm{e}^{\mathrm{i} \epsilon \mu T_{\zeta_{1}}}-1\right) \prod_{j=2,3} \prod_{p=1,2} \int_{0}^{\infty} \mathrm{d} T_{\zeta_{j}}^{p} \mathrm{e}^{-\mu(1-\mathrm{i} \epsilon) T_{\zeta_{j}}^{p}} \\
& \times \int \mathrm{d} s \mathrm{~d} u \frac{\mathrm{e}^{-\mu(1-2 \mathrm{i} \epsilon) t_{\text {enc }}(s, u)} \mathrm{e}^{\frac{\mathrm{i}}{\hbar} s u}}{\Omega t_{\text {enc }}(s, u)}
\end{aligned}
$$

where $T_{\zeta}^{p}$ refers to the duration of $p$-th part of trajectory $\zeta$ and the power of $M$ came from the 5 choices of the remaining channels. Evaluating the integrals we get

$$
D_{i_{1}=i_{2}}^{\left(2^{2}\right)}(\epsilon, 3)=\frac{-\mathrm{i} \epsilon(1-2 \mathrm{i} \epsilon)}{(1-\mathrm{i} \epsilon)^{5}} .
$$

If on the other hand, we consider the contribution of the diagram in Figure 2r, we notice that $i_{1}=i_{2}=i_{3}$, thus activating three Kronecker deltas. The contribution of this 
diagram is thus

$D_{i_{1}=i_{2}=i_{3}}^{\left(3^{1}\right)}(\epsilon, 3)=\frac{M^{3}}{T_{\mathrm{H}}^{3}} \prod_{j=1}^{3} \int_{0}^{\infty} \mathrm{d} T_{\zeta_{j}} \mathrm{e}^{-\mu T_{\zeta_{j}}}\left(\mathrm{e}^{\mathrm{i} \epsilon \mu T_{\zeta_{j}}}-1\right)=\frac{(\mathrm{i} \epsilon)^{3}}{(1-\mathrm{i} \epsilon)^{3}}$.

Comparing the results in (38), (44) and (45) we can surmise that the power of i $\epsilon$ that results from sliding an encounter into the incoming lead is equal to the number of direct stretches from the encounter to the outgoing lead, i.e. the stretches that do not participate in any other encounters. This observation can be mathematically verified by using the relationship between diagrams and factorisations of the cyclic permutation [30.

To summarise, we can write down the contribution of each diagram by simply looking at its links and encounters and assigning

- a factor of $(1-\mathrm{i} \epsilon)^{-1}$ to each stretch,

- a factor of $-(1-\mathrm{i} l \epsilon)$ to each non-degenerate $l$-encounter,

- a factor of $(\mathrm{i} \epsilon)^{s}$ to each encounter happening in the incoming lead and having $s$ direct stretches to the outgoing lead.

- a factor of 1 to each encounter happening in the outgoing lead.

Altogether, the leading contribution to $D(\epsilon, 3)$ is thus

$$
\begin{aligned}
D(\epsilon, 3)= & 3\left(\frac{(1-2 \mathrm{i} \epsilon)^{2}}{(1-\mathrm{i} \epsilon)^{7}}+\frac{-(1-2 \mathrm{i} \epsilon)}{(1-\mathrm{i} \epsilon)^{5}}+\frac{-\mathrm{i} \epsilon(1-2 \mathrm{i} \epsilon)}{(1-\mathrm{i} \epsilon)^{5}}+\frac{\mathrm{i} \epsilon}{(1-\mathrm{i} \epsilon)^{3}}\right) \\
& +\frac{-(1-3 \mathrm{i} \epsilon)}{(1-\mathrm{i} \epsilon)^{6}}+\frac{1}{(1-\mathrm{i} \epsilon)^{3}}+\frac{(\mathrm{i} \epsilon)^{3}}{(1-\mathrm{i} \epsilon)^{3}},
\end{aligned}
$$

where the factor of three counts the three different ways to label the diagram of Figure $2 \mathrm{~b}$ and its descendants.

The third moment can thus be calculated to be

$$
m_{3}=\frac{6}{\mu^{3}}
$$

and we note that if we put in three different energy arguments in line with (10), we can use the same diagrams to get the next three moments using (11) and (12) as

$$
m_{4}=\frac{22}{\mu^{4}}, \quad m_{5}=\frac{90}{\mu^{5}}, \quad m_{6}=\frac{394}{\mu^{6}} .
$$

\section{All moments}

Now that we know the rules which govern the contributions of individual diagrams, we can look at generating all the diagrams and their contributions recursively. To leading order in inverse channel number, the contributing diagrams of the type $2^{v_{2}} 3^{v_{3}} \cdots$ are in a bijective correspondence with rooted plane trees [22] that have $v_{l}$ vertices of degree $2 l$ and all other vertices of degree one (called "leaves"), see Figure 3 We denote by $V=v_{2}+v_{3}+\ldots$ the total number of vertices of degree higher than 1 . These vertices correspond to encounters in the diagram. The total number of leaves can easily be seen to be $2(L-V+1)$, where $L=2 v_{2}+3 v_{3}+\ldots$. Starting from the root the leaves are labelled $i_{1}, o_{1}, i_{2}, o_{2}, \ldots, i_{n}, o_{n}$, where $n=L-V+1$ is the order of the correlation function $D(\epsilon, n)$. The $i$-labelled leaves correspond to incoming trajectories starting in 


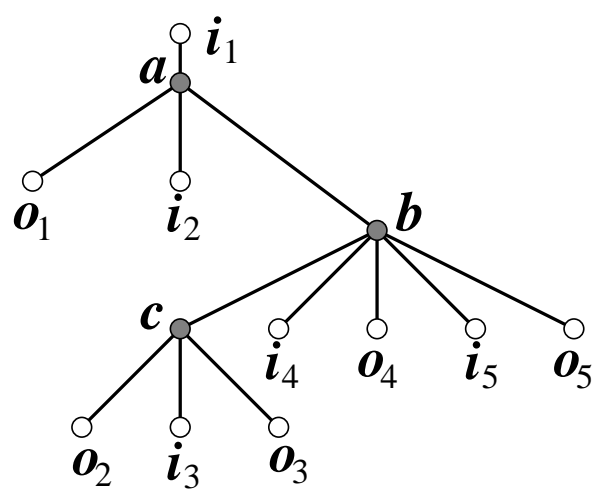

Figure 3. An example of a $\left(2^{2} 3^{1}\right)$-tree.

the lead and the $o$-labelled leaves correspond to the outgoing trajectories exiting into the lead.

As we have seen, some encounters can touch the lead, but this can only happen if an $l$-encounter has $l$ vertices with labels $i$ connected to it ( $i$-touch) or $l$ vertices with labels $o$ connected to it (o-touch). For example, in Figure 3, the vertex $a$ can $i$-touch, the vertex $c$ can $o$-touch and the vertex $b$ can do neither.

When an encounter can touch the lead, the total answer we seek is the sum of the contributions from when it does and when it does not do it. Equivalently, we can take the multiplicative factor of an encounter to be the sum of all factors it can produce. To illustrate this point, we revisit the calculation of $D(\epsilon, 3)$ and rewrite equation (46) in the form

$$
\begin{aligned}
D(\epsilon, 3)= & \frac{3}{(1-\mathrm{i} \epsilon)^{3}}\left(\frac{-(1-2 \mathrm{i} \epsilon)}{(1-\mathrm{i} \epsilon)^{2}}+1\right)\left(\frac{-(1-2 \mathrm{i} \epsilon)}{(1-\mathrm{i} \epsilon)^{2}}+\mathrm{i} \epsilon\right) \\
& +\frac{1}{(1-\mathrm{i} \epsilon)^{3}}\left(\frac{-(1-3 \mathrm{i} \epsilon)}{(1-\mathrm{i} \epsilon)^{3}}+1+(\mathrm{i} \epsilon)^{3}\right) .
\end{aligned}
$$

This is the sum of contributions of three $\left(2^{2}\right)$-trees and one $\left(3^{1}\right)$-tree, see Figure 4 . The structure of a contribution is as follows: the prefactor is $(1-\mathrm{i} \epsilon)^{-n}$, where $n$ is half the number of leaves (the order of the correlation function). Then follow the factors corresponding to the vertices of the diagram. Each vertex of degree $2 l$ (corresponding to an $l$-encounter) gives a multiplicative factor of

$$
\frac{-(1-\mathrm{i} l \epsilon)}{(1-\mathrm{i} \epsilon)^{l}}
$$

modified by an additional +1 if the vertex can $o$-touch and by $+(\mathrm{i} \epsilon)^{s}$ if the vertex can $i$-touch, where $s$ is the number of $o$-leaves attached to the vertex. In the case when there is only one vertex in the diagram, as in the $\left(3^{1}\right)$-tree in Figure 4, it can both $i$-touch and $o$-touch but not at the same time. To provide a further example, the overall contribution of the tree in Figure 3 is

$$
\frac{1}{(1-\mathrm{i} \epsilon)^{5}}\left(\frac{-(1-2 \mathrm{i} \epsilon)}{(1-\mathrm{i} \epsilon)^{2}}+\mathrm{i} \epsilon\right)\left(\frac{-(1-3 \mathrm{i} \epsilon)}{(1-\mathrm{i} \epsilon)^{3}}\right)\left(\frac{-(1-2 \mathrm{i} \epsilon)}{(1-\mathrm{i} \epsilon)^{2}}+1\right) .
$$

To count all possible trees while keeping track of the structure of their vertices we introduce the generating function $F\left(\vec{x}, \overrightarrow{z_{o}}, \sigma, \overrightarrow{z_{i}}, \tau\right)$. The roles of the variables are as follows: 

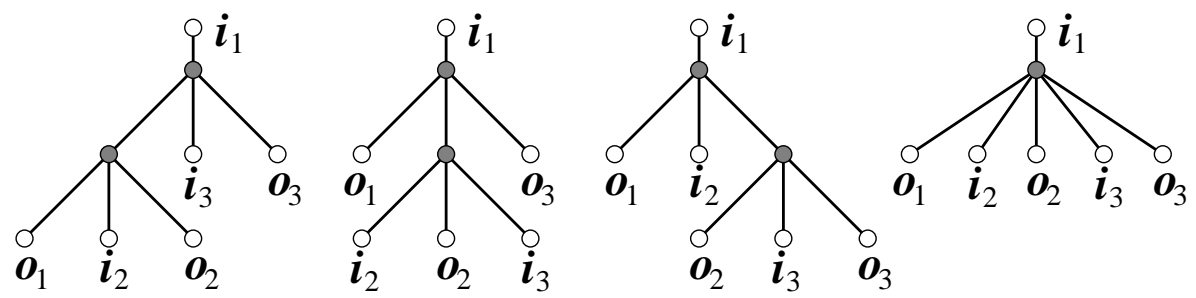

Figure 4. The trees contributing to the third order correlation function $D(\epsilon, 3)$. The first three trees correspond to relabellings of Figure $2 \mathrm{~b}$ and the last one to Figure 2.

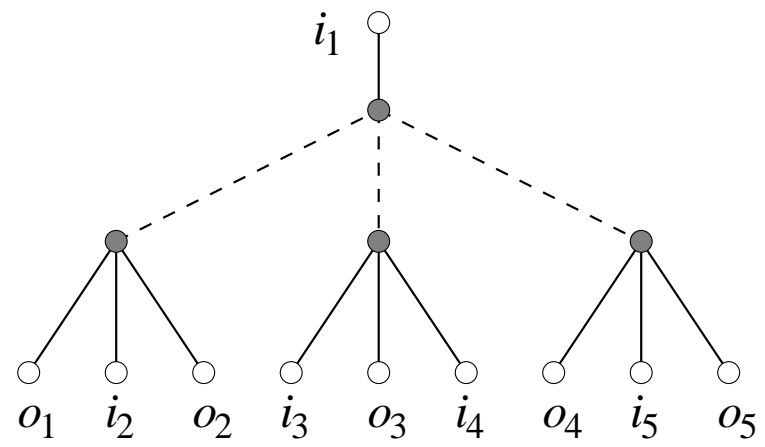

Figure 5. A separation of a tree into the top vertex and subtrees. Note that in the middle subtrees the roles of variables $\left(\overrightarrow{z_{o}}, \sigma\right)$ and $\left(\overrightarrow{z_{i}}, \tau\right)$ are reversed.

- the power of $x_{l}$ enumerates the number of non-degenerate $l$-encounters

- the power of $z_{o, l}$ enumerates the number of $l$-encounters that $o$-touch the lead

- the power of $1+\sigma$ is the total number of $i$-labelled leaves adjacent to the encounters that $o$-touch the lead

- the power of $z_{i, l}$ enumerates the number of $l$-encounters that $i$-touch the lead

- the power of $1+\tau$ is the total number of $o$-labelled leaves adjacent to the encounters that $i$-touch the lead.

For example, the tree in Figure 3 gives rise to four contributions to the generating function, corresponding to the four possibilities of the vertices $a$ and $c$ touching the lead or not

$$
\begin{gathered}
x_{2} x_{3} x_{2}+z_{i, 2}(1+\tau) x_{3} x_{2}+x_{2} x_{3} z_{o, 2}(1+\sigma)+z_{i, 2}(1+\tau) x_{3} z_{o, 2}(1+\sigma) \\
=\left(x_{2}+z_{i, 2}(1+\tau)\right) x_{3}\left(x_{2}+z_{o, 2}(1+\sigma)\right) .
\end{gathered}
$$

Our aim then is to set

$x_{l}=\frac{-(1-\mathrm{i} l \epsilon)}{(1-\mathrm{i} \epsilon)^{l}}, \quad z_{o, l}=z_{i, l}=1, \quad \sigma=0, \quad \tau=-1+i \epsilon$,

in line with the semiclassical contributions described above, and finally include a change of variables to provide the correct prefactor of $(1-\mathrm{i} \epsilon)^{-n}$.

While the generating function $F$ is our aim, we will first deal with an auxiliary function $f=f\left(\vec{x}, \overrightarrow{z_{o}}, \sigma, \overrightarrow{z_{i}}, \tau\right)$ which is defined exactly as $F$ except for not allowing the top vertex to $i$-touch the lead and not counting the root as a leaf when the top vertex 
$o$-touches the lead. These restrictions on the function $f$ makes it possible to find a recursive equation for it. The value corresponding to an empty tree will be set to 1 , i.e. $f(0)=1$. The correlation function calculated with the above restrictions will be denoted by $\tilde{D}(\epsilon, n)$.

To write a recursion for $f$ we separate a tree into the top vertex of degree $2 l$ and $2 l-1$ subtrees, see Figure [5. If the top vertex is non-degenerate, its contribution is $x_{l} f^{l} \hat{f}^{l-1}$, where the function $\hat{f}$ correspond to the even-numbered subtrees in which the positions of $i$ 's and $o$ 's are reversed. Thus the roles of all the variables corresponding to leaves of one type are switched, i.e. $\hat{f}=f\left(\vec{x}, \overrightarrow{z_{i}}, \tau, \overrightarrow{z_{o}}, \sigma\right)$.

To account for the possibility of the top vertex touching the lead, we recall that from the definition of $f$, it is only allowed to $o$-touch. In this case all odd-numbered subtrees must be empty and the contribution of each even-numbered subtree is $\hat{f}+\tau$. Note that if the subtree is empty, the contribution evaluates to the correct value of $\hat{f}(0)+\tau=1+\tau$. Putting this together, we have

$$
f=1+\sum_{l=2}^{\infty}\left[x_{l} f^{l} \hat{f}^{l-1}+z_{o, l}(\hat{f}+\tau)^{l-1}\right],
$$

and correspondingly

$$
\hat{f}=1+\sum_{l=2}^{\infty}\left[x_{l} \hat{f}^{l} f^{l-1}+z_{i, l}(f+\sigma)^{l-1}\right] .
$$

To obtain the function $F$ we need to take into account the special role of the top vertex. It can both $i$-touch and $o$-touch although not at the same time. Additionally, it is always adjacent to an $i$-labelled leaf and thus always contributes to the power of $1+\tau$. The final generating function $F$ satisfies

$$
\begin{aligned}
F & =1+\sigma+\tau+\sum_{l=2}^{\infty}\left[x_{l} f^{l} \hat{f}^{l-1}+z_{o, l}(\hat{f}+\tau)^{l-1}(1+\tau)+z_{i, l}(f+\sigma)^{l}\right] \\
& =f+\sigma+\tau+\sum_{l=2}^{\infty}\left[\tau z_{o, l}(\hat{f}+\tau)^{l-1}+z_{i, l}(f+\sigma)^{l}\right] \\
& =\sum_{l=1}^{\infty}\left[\tau z_{o, l}(\hat{f}+\tau)^{l-1}+\sigma z_{i, l}(f+\sigma)^{l-1}+f z_{i, l}(f+\sigma)^{l-1}\right],
\end{aligned}
$$

where, to get to the last line we defined $z_{o, 1}=z_{i, 1}=1$. We note that the value $F(0)$ cannot be defined from recursive considerations and needs to be chosen to provide the correct answer for $D(\epsilon, 1)$, which turns out to be $\mathrm{i} \epsilon=1+\sigma+\tau$. Another important observation is that our choice of the leaf $i_{1}$ as the root (see Figure 3 for example) is arbitrary. In particular an o-leaf could be chosen and the answer for $D(\epsilon, n)$ should not depend on the choice. Thus the function $F$ should be symmetric with respect to swapping the variables $z_{o}$ with $z_{i}, \tau$ with $\sigma$ and $f$ with $\hat{f}$. This is not apparent from (56) but will be checked (and used!) at a later stage.

Now we can make the substitutions

$$
\begin{aligned}
& x_{l}=-\frac{1-\mathrm{i} l \epsilon}{(1-\mathrm{i} \epsilon)^{l}} \tilde{r}^{l-1}, \\
& z_{o, l}=z_{i, l}=\tilde{r}^{l-1}, \\
& \tilde{r}=\frac{r}{(1-\mathrm{i} \epsilon)}, \\
& \sigma \quad=0, \quad \tau=-1+\mathrm{i} \epsilon .
\end{aligned}
$$


The substitutions give the contribution of each tree diagram as in (53), but we have included the powers of $\tilde{r}$ to keep track of which order correlation function they contribute to. Indeed, the power of $\tilde{r}$ corresponding to a $2^{v_{2}} 3^{v_{3}} \ldots$ tree would be $v_{2}(2-1)+v_{3}(3-1)+\ldots=L-V=n-1$. Substitution (59) therefore gives a prefactor of $(1-\mathrm{i} \epsilon)^{1-n}$ and so to get the additional factor of $(1-\mathrm{i} \epsilon)^{-1}$ we need for the correct prefactor, we introduce $g=(1-\mathrm{i} \epsilon)^{-1} f$. The function $g$ is a generating function of the "restricted" coefficients $\tilde{D}(\epsilon, n)$, i.e.

$$
g=\sum_{n=1}^{\infty} r^{n-1} \tilde{D}(\epsilon, n)
$$

Performing all the changes of variable, apart from (60) for now, from (54) we arrive at

$g(1-\mathrm{i} \epsilon)=1-\sum_{l=2}^{\infty} r^{l-1} g^{l} \hat{g}^{l-1}(1-\mathrm{i} l \epsilon)+\sum_{l=2}^{\infty} r^{l-1}\left(\hat{g}+\frac{\tau}{(1-\mathrm{i} \epsilon)}\right)^{l-1}$,

and a similar equation for $\hat{g}$. The sums can be performed easily, especially when we notice that the first two terms correspond to the $l=1$ terms of the two sums, leading to

$$
\frac{g}{1-r g \hat{g}}=\frac{\mathrm{i} \epsilon g}{(1-r g \hat{g})^{2}}+\frac{1}{1-r \hat{g}-\frac{r \tau}{(1-\mathrm{i} \epsilon)}} .
$$

Taking the numerator of the above equation, we arrive at

$$
(1-\mathrm{i} \epsilon-r g \hat{g})\left[\frac{1+r g \tau}{1-\mathrm{i} \epsilon}-g\right]+(\mathrm{i} \epsilon)^{2} r g \hat{g}=0,
$$

and similarly for $\hat{g}$

$$
(1-\mathrm{i} \epsilon-r g \hat{g})\left[\frac{1+r \hat{g} \sigma}{1-\mathrm{i} \epsilon}-\hat{g}\right]+(\mathrm{i} \epsilon)^{2} r g \hat{g}=0 .
$$

We have written the equations in a form that highlights the symmetric terms involving $g \hat{g}$. Taking the difference between (64) and (65) we obtain

$$
g(1-\mathrm{i} \epsilon-r \tau)=\hat{g}(1-\mathrm{i} \epsilon-r \sigma)
$$

This can now be substituted back into (64) or (65) to give an implicit equation for $g$ or $\hat{g}$, although we only state the result after the simplifying substitution from (60)

$$
\left[g-\frac{1}{(1-\mathrm{i} \epsilon)}\right](1+r)=\frac{r g^{2}}{(1-\mathrm{i} \epsilon)}\left[g-\frac{1}{(1-\mathrm{i} \epsilon)}-\frac{\epsilon^{2}}{(1-\mathrm{i} \epsilon)}\right],
$$

Putting all substitutions apart from (60) into (56), and defining $G=r(1-\mathrm{i} \epsilon)^{-1} F$, we obtain

$$
\frac{G}{r}=\frac{g}{1-r g-\frac{r \sigma}{(1-\mathrm{i} \epsilon)}}+\frac{\sigma}{(1-r g)(1-\mathrm{i} \epsilon)-r \sigma}+\frac{\tau}{(1-r \hat{g})(1-\mathrm{i} \epsilon)-r \tau} .
$$

Though it is clear that the last two terms together are symmetric, this still needs to be checked for the first term. We can rewrite it as

$\frac{g}{1-r g-\frac{r \sigma}{(1-\mathrm{i} \epsilon)}}=\frac{g(1-\mathrm{i} \epsilon)}{1-\mathrm{i} \epsilon-r \sigma-r g(1-\mathrm{i} \epsilon)}=\frac{g \hat{g}(1-\mathrm{i} \epsilon)}{\hat{g}(1-\mathrm{i} \epsilon-r \sigma)-r g \hat{g}(1-\mathrm{i} \epsilon)}$,

so that we can see the symmetry follows from (66).

Having verified the symmetry of the generating function $G$ we can put in the symmetry-breaking substitution (60), or rather the simpler and equivalent $\tau=0$ and 
$\sigma=\mathrm{i} \epsilon-1$. We finally get the generating function of the required semiclassical correlation functions

$$
G=\frac{r(g-1)}{1-r(g-1)}, \quad G=\sum_{n \geq 1} r^{n} D(\epsilon, n) .
$$

From (15), we can see that the $n$-th moment is simply $(\mathrm{i} \mu)^{-n}$ times the coefficient of $(\epsilon r)^{n}$ in the expansion of $G$. Setting $a=\mathrm{i} \epsilon$ to cancel the factors of i, we just need to extract the coefficients of $(a r)^{n}$ from $G$. To do so we let $y=r(g-1)$ and obtain

$$
G=\frac{y}{1-y}, \quad y=\frac{G}{1+G} .
$$

Substituting $g=1+y / r, r=s / a$ and setting $a=0$ in equation (67), we get the equation

$$
s-y s-y+2 y^{2}=0,
$$

for $y$ and, through relation (71), the equation

$$
G^{2}+(s-1) G+s=0,
$$

for the generating function of the moments. Solving this, and taking the solution which gives the correct value of $G=0$ when $r=0$, we get exactly the generating function (25) from RMT. As the semiclassically calculated moments match the RMT ones to all orders, we then recover the full distribution of the delay times (18) from [17.

\section{Conclusions and Outlook}

For open chaotic systems we presented a semiclassical derivation of all moments of the individual delay times, up to leading order in inverse channel number. The derivation essentially relies on the twin properties of hyperbolicity and ergodicity of the chaotic dynamics: the latter for the possible return to nearby points to create encounters and the former to allow the reconnections inside those encounters. This leads to a result which is in agreement with the RMT prediction for the delay time distribution, a semicircle type law. Notably this implies an upper bound on the longest delay time. In the derivation we relied heavily on the previous work on semiclassical expansions, in particular [19, 21, which contain an implicit assumption of instant equilibration in the underlying classical dynamics. The influence of slower equilibration can be explored by treating the effect of the Ehrenfest time on the semiclassical contributions 23, 31. When the Ehrenfest time becomes much larger than the typical time trajectories spend inside the system one recovers the classical exponential distribution of delay times.

It is also interesting to explore the effect of moving from chaotic to mixed phase space (with regular islands) on the distribution of the delay times and this upper bound. The interference effects which lead to the current result rely on the chaotic dynamics and should be suppressed if the chaotic part is reduced. However, additional effects such as periodic orbit bifurcations 32 can also be fairly strong. The moments of the delay times and in particular their upper bound could therefore be very sensitive measures to explore the dynamics inside quantum dots, and possibly used to measure the relative weights of the chaotic and regular parts of phase space.

By considering scattering matrix correlation functions, which were then treated semiclassically, we were able to derive equations that implicitly define the generating functions of the moments. It is worth noting here that such correlation functions are useful for investigating other questions, such as the density of states of chaotic Andreev billiards. 
Restricting our attention to the leading order in inverse channel number resulted in limiting the contributing diagrams to trees only. This, in turn, allowed standard recursive tools to be used. Looking beyond the leading order, the semiclassical diagrams have more complicated structures so that such tools can no longer be directly applied. Thus, in our view, the remaining challenge of obtaining a semiclassical expansion of all moments to all orders is a task of significant technical difficulty. However, it is a task of particular interest, not only because the subleading orders should be influenced by the symmetries of the system, but also because the effects of a finite number of channels is of much experimental relevance. A solution to this problem would have to involve new combinatorial tools and we hope that a clear and general algebraic structure will emerge as a result of research in this direction.

\section{Acknowledgments}

The authors would like to thank Cyril Petitjean, Misha Polianski and Daniel Waltner for useful discussions and gratefully acknowledge the Alexander von Humboldt Foundation (JK) and the National Science Foundation under Grant No. 0604859 (GB) for funding.

\section{Appendix A. Correlation coefficients $C(\epsilon, n)$}

Here we briefly outline the results for the correlation functions $C(\epsilon, n)$ defined in (17). As these do not involve subtracting the identity matrix from each bracket (as we did in (13)) we no longer need to change the contribution of certain diagonal pairs or subtract anything when the start channels coincide. We can therefore simply set both $\sigma=\tau=0$ in the treatment of section 4 and generate $C(\epsilon, n)$ instead. This simplification means that $g=\hat{g}$ because of (66), so that $g$ is now given implicitly by

$$
\left[g-\frac{1}{(1-\mathrm{i} \epsilon)}\right]=\frac{r g^{2}}{(1-\mathrm{i} \epsilon)}\left[g-\frac{1}{(1-\mathrm{i} \epsilon)}-\frac{\epsilon^{2}}{(1-\mathrm{i} \epsilon)}\right],
$$

where in fact the only difference is that the $r$ on the left hand side of (67) has disappeared. We can generate the first few terms in the expansion $g=\sum r^{n-1} g_{n}$ as

$$
g_{0}=\frac{1}{(1-\mathrm{i} \epsilon)}, \quad g_{1}=\frac{-\epsilon^{2}}{(1-\mathrm{i} \epsilon)^{4}}, \quad g_{2}=\frac{\epsilon^{2}\left(2 \epsilon^{2}-1\right)}{(1-\mathrm{i} \epsilon)^{7}} .
$$

Adding the contribution from the top node in $F$, we obtain the full generating function of $C(\epsilon, n)$, which is given by

$$
G(\epsilon, r)=\frac{r g}{1-r g}=r g+r^{2} g^{2}+\ldots
$$

The expansion $G(\epsilon, r)=\sum r^{n} C(\epsilon, n)$ then leads to

$$
\begin{aligned}
& C(\epsilon, 1)=g_{0}=\frac{1}{(1-\mathrm{i} \epsilon)}, \\
& C(\epsilon, 2)=\left[g_{1}+g_{0}^{2}\right]=\frac{1-2 \mathrm{i} \epsilon-2 \epsilon^{2}}{(1-\mathrm{i} \epsilon)^{4}}, \\
& C(\epsilon, 3)=\left[g_{2}+2 g_{1} g_{0}+g_{0}^{3}\right]=\frac{1-4 \mathrm{i} \epsilon-9 \epsilon^{2}+8 \mathrm{i} \epsilon^{3}+5 \epsilon^{4}}{(1-\mathrm{i} \epsilon)^{7}},
\end{aligned}
$$

which are exactly the correlation functions we can obtain by considering the diagrams explicitly as in section 3 . Combining these results in line with expanding (13), we 
recover the first three functions $D(\epsilon, n)$ calculated in section 3 . We could also continue to generate terms to obtain the moments via (16).

However, the function $G(\epsilon, r)$ contains more information than just the moments, for example by setting $\epsilon=0$ we see that $g=1$. Hence

$$
G(0, r)=\frac{r}{1-r}=r+r^{2}+\ldots
$$

which shows that

$$
\operatorname{Tr}\left[S^{\dagger}(E) S(E)\right]^{n}=M,
$$

and that the unitarity of the scattering matrix holds semiclassically for all powers $n$ to leading order in inverse channel number. As another example, this function $G$ and the correlation functions it generates appear in the density of states of chaotic Andreev billiards 23.

\section{References}

[1] L. Eisenbud 1948 PhD thesis, Princeton

[2] E. P. Wigner 1955 Phys. Rev., 98 145-147

[3] W. O. Amrein and Ph. Jacquet 2007 Phys. Rev. A, 75022106

[4] F. T. Smith 1960 Phys. Rev., 118 349-356

[5] C. H. Lewenkopf and R. O. Vallejos 2004 Phys. Rev. E, 70036214

[6] J. Kuipers and M. Sieber 2008 Phys. Rev. E, 77046219

[7] R. Balian and C. Bloch 1974 Ann. Phys., 85 514-545

[8] R. O. Vallejos, A. M. Ozorio de Almeida and C. H. Lewenkopf 1998 J. Phys. A, 31 4885-4897

[9] M. C. Gutzwiller 1971 J. Math. Phys., 12 343-358

[10] M. C. Gutzwiller 1990 Chaos in classical and quantum mechanics Springer, New York

[11] M. V. Berry 1985 Proc. Roy. Soc. A, $400229-251$

[12] J. H. Hannay and A. M. Ozorio de Almeida 1984 J. Phys. A, 17 3429-3440

[13] M. Sieber and K. Richter 2001 Phys. Scr., T90 128-133

[14] S. Müller, S. Heusler, P. Braun, F. Haake and A. Altland 2004 Phys. Rev. Lett., 93014103

[15] S. Müller, S. Heusler, P. Braun, F. Haake and A. Altland 2005 Phys. Rev. E, 72046207

[16] J. Kuipers and M. Sieber 2007 Nonlinearity, 20 909-926

[17] P. W. Brouwer, K. M. Frahm and C. W. J. Beenakker 1999 Waves in Random Media, $991-104$

[18] K. Richter and M. Sieber 2002 Phys. Rev. Lett., 89206801

[19] S. Heusler, S. Müller, P. Braun and F. Haake 2006 Phys. Rev. Lett., 96066804

[20] P. Braun, S. Heusler, S. Müller and F. Haake 2006 J. Phys. A, 39 L159-L165

[21] S. Müller, S. Heusler, P. Braun and F. Haake 2007 New J. Phys., 912

[22] G. Berkolaiko, J. M. Harrison and M. Novaes 2008 J. Phys. A, 41365102

[23] J. Kuipers, D. Waltner, C. Petitjean, G. Berkolaiko and K. Richter 2009 Phys. Rev. Lett. in press, arXiv:0907.2660v2

[24] C. H. Lewenkopf and R. O. Vallejos 2004 J. Phys. A, 37 131-136

[25] D. Gouyou-Beauchamps and B. Vauquelin 1988 RAIRO Inform. Théor. Appl., 22 361-388

[26] N. J. A. Sloane 2009 The on-line encyclopedia of integer sequences, published electronically at http://www.research.att.com/ njas/sequences/

[27] W. H. Miller 1975 Adv. Chem. Phys., 30 77-136

[28] K. Richter 2000 Semiclassical theory of mesoscopic quantum systems Springer, Berlin

[29] H. Schanz, M. Puhlmann and T. Geisel 2003 Phys. Rev. Lett., 91134101

[30] G. Berkolaiko, J. M. Harrison and M. Novaes 2008 Preprint, arXiv:0809.3476

[31] D. Waltner et al. 2010 in preparation

[32] J. P. Keating, A. M. Ozorio de Almeida, S. D. Prado, M. Sieber and R. Vallejos 2007 Prog. Theor. Phys. Suppl., 166 10-18 